\title{
Discussion
}

Aller: What was the scale and resolution of the photographs used for M33?

Courtès: I have used three different kinds of exposure. I took the diameter of three different stars of different magnitude for correction of the diameter of the HII regions. The resolution is no more than $4^{\prime \prime}$ of arc, since it is reduced by a factor 5 because of the focal length of the instrument.

Feast: Are the motions you measure in the outer parts of the 30 Doradus nebula systematic or not?

Courtès: They are not really systematic; I have a list of radial velocities available which there is not time to present.

\section{RADIO CONTINUUM EMISSION FROM HII REGIONS IN THE MAGELLANIC CLOUDS}

\author{
D. S. Mathewson and J. R. Healey \\ CSIRO Radiophysics Laboratory, Sydney
}

\section{Introduction}

A considerable proportion of the radio emission at high frequencies from the Galaxy is of thermal origin. This thermal radiation appears to originate both in very extended regions and in discrete sources. It has always been difficult to compare the radio and optical data for these HII regions as galactic optical observations are hampered by heavy obscuration. However, the HII regions in the Magellanic Clouds are relatively free from obscuration, and spectrophotometry by Dickel, Aller, and Faulkner (this volume, paper 63) and Henize (1956) have provided very good measurements of $\mathrm{H} \beta$ and $\mathrm{H} \alpha$ flux densities and the relative intensities of the OII, OIII lines. Also the distance to the Clouds is fairly well known (about $55 \mathrm{kpc}$ ) so that the linear dimensions of the HII regions may be estimated with some degree of accuracy in contrast to the galactic emission nebulae.

The high resolving power and sensitivity of the 210 -foot reflector of the Australian National Radio Astronomy Observatory at high frequencies provide for the first time an opportunity to compare the radio and optical data for the HII regions in the Clouds. Observations were carried out at 1410 and $2650 \mathrm{Mc} / \mathrm{s}$ where the aerial beamwidths at half-intensity points are $14^{\prime}$ of arc and $7: 5$ of arc respectively and the receiving systems allow temperature differences of $0 \cdot 1^{\circ} \mathrm{K}$ to be measured. The 1410 $\mathrm{Mc} / \mathrm{s}$ isophotes were presented in a previous session (paper 54) at this Symposium on "The Continuum Radio Emission from the Magellanic Clouds". The extended and discrete thermal emitting regions in the Large and Small Magellanic Clouds will now be discussed and compared with galactic HII regions. Throughout this discussion it will be assumed that the electron temperature of HII regions is $10^{4} \mathrm{~K}$.

\section{Extended Thermal Regions}

From the results of the 2650,1410 , and $408 \mathrm{Mc} / \mathrm{s}$ surveys of the Large Cloud (Figs. 2, 3, and 4 in paper 54) and using $-0 \cdot 6$ for the spectral index of the nonthermal emission, it was possible to delineate an extended thermal region centred on R.A. 
$05^{\mathrm{h}} 40^{\mathrm{m}}$, dec. $-69^{\circ} 30^{\prime}$. The size at half-intensity points was R.A. $2^{\circ}$ by dec. $3^{\circ}$ and the integrated emission was about 40 flux units (one flux unit equals $10^{-26} \mathrm{Wm}^{-2}(\mathrm{c} / \mathrm{s})^{-1}$ ). It was the only very extensive HII region detected in the Large Cloud.

In the Galaxy, the most outstanding extended thermal region appears to lie symmetrically distributed in longitude about the galactic centre to a radius of $4 \mathrm{kpc}$. It has a thickness in latitude of only 200 pc (Mathewson, Healey, and Rome 1962b). Calculation shows that the emission measure of this component when viewed face-on would be very similar to that obtained for the region in the Large Cloud.

The Small Magellanic Cloud appears to have coextensive with the bright optical bar an extended thermal source which causes a "flattening" of the spectral index at the higher frequencies. Its emission measure is about 200. As the Small Cloud is viewed edge-on, it may be due to a number of unresolved thermal discrete sources.

\section{Discrete HII Regions}

(a) Optically Unidentified "Thermal" Radio Sources in the Outskirts of the Magellanic Clouds.-The $1410 \mathrm{Mc} / \mathrm{s}$ isophotes of the Large and Small Clouds (see paper 54) present the most detailed radio picture obtained so far for external galaxies. The majority of the discrete radio sources lying in the main body of the Clouds (i.e. within contour No. 2) have been identified with emission nebulae listed in Henize's catalogue. In the outskirts of the Clouds, a number of radio sources have been detected but no optical identifications have been made. On using the source statistics of Mills, Slee, and Hill (1960), there appears to be a normal concentration of sources in this region surrounding the Clouds and therefore they are probably "general field" radio sources and unassociated with the Clouds. However, to gain more information about these sources, flux density spectral index measurements were made between 1410,408 , and where possible $85.5 \mathrm{Mc} / \mathrm{s}$. Most of the sources were nonthermal but $10 \%$ had a spectral index $(\alpha)$ close to zero, which suggests a thermal origin of the radiation. The positions, sizes, and intensities of these sources with zero spectral index are listed in Table 1 for the Small and Large Clouds, and it may prove worth while to study optically these points in more detail.

(b) Optically Identified Sources in the Magellanic Clouds. $-\mathrm{H} \beta$ photographs of the Magellanic Clouds show that the larger HII associations (excluding 30 Doradus which has been discussed in paper 54) consist of a number of bright knots about 4 min of arc $(70 \mathrm{pc})$ in diameter embedded in a faint nebulous background. The overall dimension of an association is typically of the order of $12 \mathrm{~min}$ of arc. The resolution of the 210 -foot reflector at $1410 \mathrm{Mc} / \mathrm{s}(14 \mathrm{~min}$ of arc beam) and even at $2650 \mathrm{Mc} / \mathrm{s}(7.5 \mathrm{~min}$ of arc beam) is inadequate to investigate the radio emission from the individual knots in such an association. $\mathrm{H} \beta$ and $\mathrm{OII} / \mathrm{OIII}$ spectrophotometry is better for a detailed study of these regions. However, a very important test may be made by calculating the integrated radio emission expected from an association on using the $\mathrm{H} \beta$ flux densities of the individual knots from measurements of Dickel, Aller, and Faulkner (this volume, paper 63) and comparing the result with that observed. If good agreement is obtained the radio emission is of thermal origin. If more radio emission is observed than predicted from the $\mathrm{H} \beta$ measurements some of the radio emission may be of nonthermal origin (some disagreement is to be expected owing to errors in the inter- 
stellar reddening correction and in the radio flux density measurements). This is an excellent method of searching for supernovae, some of which are strong radio emitters, such as Cassiopeia A and the Crab nebula in the Galaxy. Spectral index measurements are not such a good guide for distinguishing between thermal and nonthermal sources as some galactic "radio" supernovae have spectral indices very similar to that of an "optically thin" thermal source. Also the two frequencies, $1410 \mathrm{Mc} / \mathrm{s}$ and $2650 \mathrm{Mc} / \mathrm{s}$, used to study these HII associations, are so close that spectral index measurements are unreliable.

TABLE 1

UNIDENTIFIED SOURCES WITH $\alpha=0$ SURROUNDING THE SMALL AND LARGE MAGELLANIC CLOUDS

\begin{tabular}{|c|c|c|c|c|c|}
\hline \multicolumn{4}{|c|}{ Position (1950) } & \multirow{2}{*}{$\begin{array}{c}\text { Size } \\
(\min \text { of } \operatorname{arc})\end{array}$} & \multirow{2}{*}{$\begin{array}{l}1410 \mathrm{Mc} / \mathrm{s} \text { Flux Density } \\
\times 10^{-26}\left(\mathrm{Wm}^{-2}(\mathrm{c} / \mathrm{s})^{-1}\right)\end{array}$} \\
\hline \multicolumn{2}{|c|}{$\begin{array}{l}\text { R.A. } \\
\text { h }\end{array}$} & \multicolumn{2}{|c|}{$\begin{array}{c}\text { Dec. (S) } \\
\circ\end{array}$} & & \\
\hline 00 & $20 \cdot 5$ & 74 & 44 & 7 & $1 \cdot 0$ \\
\hline & $37 \cdot 0$ & 68 & 49 & $20 \times 10$ & $1 \cdot 2$ \\
\hline 00 & $38 \cdot 5$ & 72 & 00 & 13 & $1 \cdot 2$ \\
\hline 00 & $53 \cdot 8$ & 71 & 22 & $\leqslant 6$ & 0.4 \\
\hline 01 & $09 \cdot 6$ & 75 & 44 & $14 \times 36$ & $1 \cdot 8$ \\
\hline 01 & $18 \cdot 1$ & 68 & 24 & $\leqslant 6$ & $1 \cdot 0$ \\
\hline 01 & $46 \cdot 0$ & 74 & 54 & $24 \times 45$ & $2 \cdot 5$ \\
\hline 04 & $36 \cdot 7$ & 74 & 54 & 11 & $1 \cdot 4$ \\
\hline 04 & $38 \cdot 6$ & 67 & 25 & $\leqslant 6$ & 0.5 \\
\hline 04 & $54 \cdot 8$ & 64 & 51 & $\leqslant 6$ & $1 \cdot 0$ \\
\hline 05 & $51 \cdot 4$ & 64 & 04 & $11 \times 17$ & $1 \cdot 2$ \\
\hline 06 & $09 \cdot 6$ & 63 & 32 & 14 & $1 \cdot 0$ \\
\hline 06 & $10 \cdot 8$ & 72 & 46 & 10 & $1 \cdot 5$ \\
\hline
\end{tabular}

Good agreement between the radio and optical observations was obtained for most of the HII regions studied except for the emission nebulae catalogued by Henize (1956) as N49, 63A, 132D in the Large Cloud and N50-52 A/B in the Small Cloud. N49 is a very interesting case. The 1410 and $408 \mathrm{Mc} / \mathrm{s}$ observations and the $85.5 \mathrm{Mc} / \mathrm{s}$ survey of Mills, Slee, and Hill (1961) show the presence of a nonthermal source in the vicinity of this region. The very high resolution of the 210 -foot reflector at $2650 \mathrm{Mc} / \mathrm{s}$ (7.5 of arc aerial beamwidth) was used to resolve this source from other nearby thermal sources. It was found that the nonthermal source coincided exactly with the position of N49 (the maximum error of the position measurement was 0.5 of arc). The source was unresolved by the 7.5 of arc aerial beam and the intensity at $2650 \mathrm{Mc} / \mathrm{s}$ was $1 \cdot 3$ flux units, much stronger than that calculated from the $\mathrm{H} \propto$ measurements. The flux density spectral index of the radiation is $-0 \cdot 8$. Henize quotes a size of $\mathbf{1}^{\prime}$ of arc for this emission nebula and comments that OI shows strong emission which is very unusual for an HII region. Dr. Westerlund has kindly provided a photograph of N49 (Fig. 1) taken with the Uppsala Schmidt 
telescope at Mt. Stromlo. There is no central exciting star and it has a looped filamentary appearance characteristic of some remnants of galactic supernovae. It is therefore suggested that $\mathrm{N} 49$ is a supernova remnant and it is the first extragalactic "radio" supernova to be discovered. Cassiopeia A, the well-known galactic supernova, which has a similar spectral index to $\mathrm{N49}$, would be five times more intense if placed at the same distance as N49 (55 kpc).

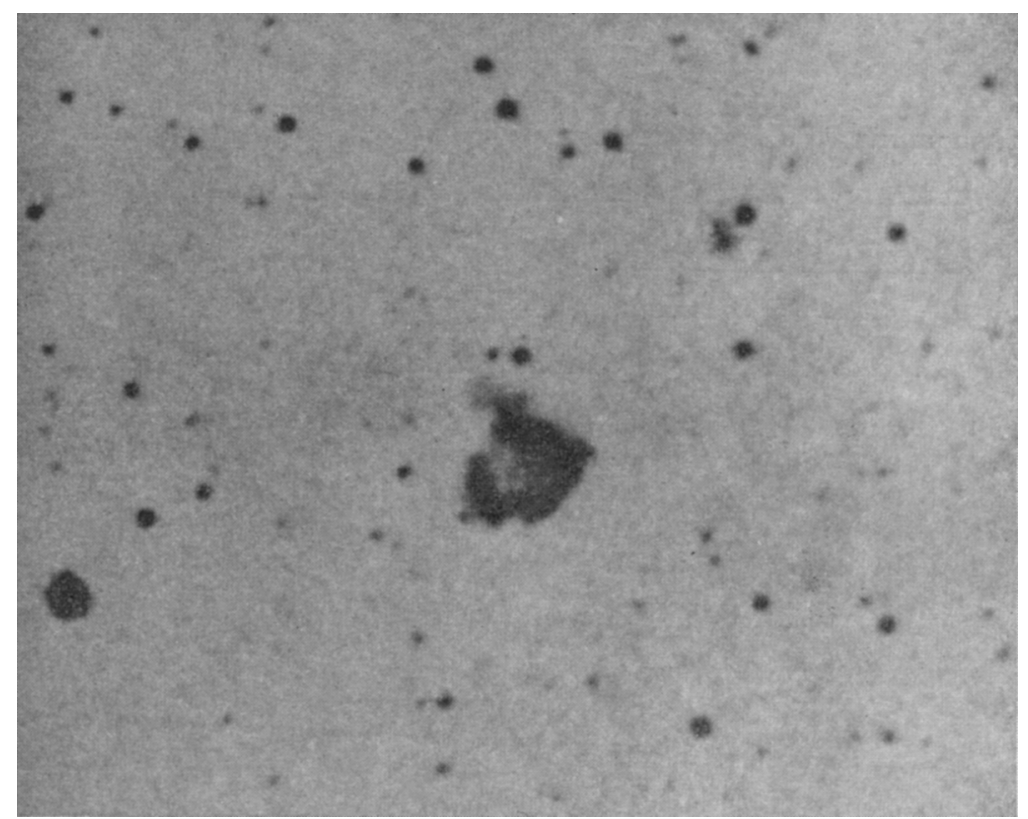

Fig. 1.-A photograph in red light of Henize N49 taken with the Uppsala Schmidt telescope at Mt. Stromlo (Dr. Westerlund).

N63A may be another supernova remnant as the radio source associated with this emission nebula is 15 times more intense than the $\mathrm{H} \beta$ measurements predict. Henize also reports OI emission from this region. However, the spectral index of the radio emission must be considerably "flatter" than that of $\mathrm{N} 49$ as the radio source does not appear very strongly at $408 \mathrm{Mc} / \mathrm{s}$.

The strongest radio source in the main body of the Large Magellanic Cloud with the exception of 30 Doradus coincides in position with N132D, a member of a small HII association. The radio emission is much more intense than expected from $\mathrm{H} \beta$ measurements, although if nonthermal radiation is present its spectral index is "flatter" than $-0 \cdot 5$. It is interesting to note that this source lies almost at the optical centroid of the axial bar and at the position of maximum emission of the extended nonthermal source associated with the axial bar (Fig. 6, Paper 54). Similarly in the Small Magellanic Cloud, a radio source coincides with a weak patchy region of emission nebulosities (N50-52) which is also close to the optical centroid of the bar. The radio emission is much stronger than that calculated from the $\mathrm{H} \beta$ measurement. 
The possibility exists that a radio source unassociated with the Magellanic Clouds may coincide with the position of an emission nebula in these galaxies. It is therefore necessary to make detailed optical investigations in an attempt to obtain more information about these objects.

\section{Comparison of HII Regions in the Magellanic Clouds and in the Galaxy}

Calculation shows that if a 60 -foot reflector were placed in the plane of the Large Magellanic Cloud about $10 \mathrm{kpc}$ from the centre and scanned along the plane, the brightness temperatures and beam profiles observed at $1410 \mathrm{Mc} / \mathrm{s}$ would be very similar to that obtained when scanning along the galactic plane (Mathewson, Healey, and Rome 1962a). It is assumed in this calculation that all the HII regions in the Large Cloud lie in a plane. This is very interesting especially as it is also noted that the emission measures for the galactic HII sources (Westerhout 1958) are about 20,000, very similar to those in the Large Cloud. Westerhout (1958) has made a number of identifications of these galactic radio sources with nearby emission nebulae. These all lie within a few kiloparsecs and are fairly small regions with diameters of about 10 pc. However, there are a number of unidentified strong radio sources with spectral indices of zero (indicative of a thermal origin of the radiation) within $60^{\circ}$ either side of the galactic centre and lying very close to the galactic plane, suggesting that they are very distant objects, e.g. Westerhout (1958) sources 30, 31, 39, 41, 42, 43, 47, 49, and 51, and Mathewson, Healey, and Rome (1962a) sources $20,36,37,42,55$, and 56. In particular, W.30, 39, and 41 and M.H.R. 36,42 , and 56 are seen in emission at $85.5 \mathrm{Mc} / \mathrm{s}$ by Hill, Slee, and Mills (1958) against background temperatures greater than $10^{4} \mathrm{~K}$. If they were nearby thermal objects with an electron temperature of $10^{4} \mathrm{~K}$, they would be seen in absorption. Hence if they are thermal regions they must be very distant. Therefore it seems quite likely that in the Galaxy there exists the same type of very massive HII associations that is found in the Magellanic Clouds. This is supported by the $1410 \mathrm{Mc} / \mathrm{s}$ observations of Hill and Komesaroff (this volume, paper 26), using the 210-foot reflector which resolve these galactic HII associations that are seen as single sources by a 60- or 80 -foot reflector, into two or three sources. This is exactly what would be expected if a typical Magellanic Cloud HII region (Section III $(b)$ ) were placed in the Galaxy at distances of $10-20 \mathrm{kpc}$. It is also interesting to note that if a typical Large Cloud HII region were placed 1-2 kpc from the Sun it would appear very similar in angular size and intensity to the Cygnus $\mathrm{X}$ complex (Mathewson, Large, and Haslam 1960).

\section{References}

Henize, K. G. (1956).-Ap. J. Supp. 2 : 315-44.

Hill, E. R., Slee, O. B., and Mills, B. Y. (1958).-Aust. J. Phys. 11 : 530-49.

Mathewson, D. S., Healey, J. R., and Rome, J. M. (1962a).-Aust. J. Phys. 15: 354-68.

Mathewson, D. S., Healey, J. R., and Rome, J. M. (1962b).-Aust. J. Phys. 15 : 369-77.

Mathewson, D. S., Large, M. I., and Haslam, C. G. T. (1960).-M.N. $120: 242-7$.

Mrlus, B. Y., Slee, O. B., and Hill, E. R. (1960).-Aust. J. Phys. 13 : 676-99.

Mrlls, B. Y., Slee, O. B., and Hill, E. R. (1961).-Aust. J. Phys. 14 : 497-507.

Westerhout, G. (1958).-B.A.N. $14: 215-60$. 


\section{Discussion}

Westerhout: The nearby HII regions are definitely small and dense clouds. Within 2 kpc there are none of the large ionized regions we see in the Magellanic Clouds. It seems also that the existence of these dense clouds close to the Sun is peculiar; if they existed say at $4 \mathrm{kpc}$ we would still easily see them radiowise. We might be in a cluster (4 kpc in diameter) of such dense clouds.

We do have faint large regions, such as the Gum nebula, but that is not dense enough to be comparable with the Magellanic Cloud nebulae. The Cygnus $\mathrm{X}$ complex is not really a good comparison either, because it derives its extent and brightness mainly from the fact that we are looking along a few kpc of spiral arm.

In view of this, I think the chance that there are large HII regions in our own Galaxy is fairly small. They would stand out on the records.

Of course, the resolving power used for the Magellanic Clouds is not large enough to find details much smaller than $300 \mathrm{pc}$. Therefore, the large sources might well consist of aggregates of small HII regions, around OB stars, embedded in a large HI region.

Mathewson: I disagree with Dr. Westerhout that "within $2 \mathrm{kpc}$ there are none of the large ionized regions we see in the Magellanic Clouds". The Cygnus X HII complex is apparently only $1.5 \mathrm{kpc}$ from the Sun. The intensity and angular size of this massive HII region is very similar to that expected from one of these king-sized Cloud HII regions if it were placed at the same distance from the Sun.

The aerial beam of the 210 -foot reflector at $2650 \mathrm{Mc} / \mathrm{s}$ allows source size measurements down to $70 \mathrm{pc}$ in the Clouds - spectrophotometrists have even better resolving power - and quite a few of the large HII regions in an association do not appear to break up into a lot of smaller ones.

Westerhout: Cygnus $\mathrm{X}$ is a typical example of how resolution influences one's conclusions. In the early days, it was one big source. Now we know that it consists of many separate, small HII regions, virtually unconnected, spread over $2 \mathrm{kpc}$ of spiral arm. One would certainly not call Cygnus X a "massive HII region". The comparison Dr. Mathewson makes is a good one, but I should like to reverse his conclusion, namely, that it points in the direction of the large regions in the Magellanic Clouds probably being aggregates of many small HII regions. Estimates of total mass therefore might be one or even several orders of magnitude too high if they are based on the assumption of one large HII region.

Mills: Firstly, I would like to point out that a decision as to whether the galactic sources of flat spectrum are superthermal, very distant giant HII regions, or supernova remnants, can probably be decided by means of high resolution observations in the neighbourhood of $30 \mathrm{Mc} / \mathrm{s}$. HII regions, even at great distances, would show marked spectral effects, in almost all cases being seen in absorption, while the frequency should be high enough to penetrate right through the extended HII regions of the Galaxy. Secondly, I would like to ask Dr. Mathewson to expand a little more on his methods of separating the Cloud sources, particularly the nonthermal sources, from the background of distant radio galaxies. This separation would seem to be quite difficult.

Mathewson: The three non-thermal sources that I associate with the Clouds all coincide in position (the accuracy of measurement is about $1 \mathrm{~min}$ of arc) with unusual objects in the Clouds. I think it is quite safe under these circumstances to identify the radio sources with these objects.

Davies: The density of radio sources outside the SMC is very similar to that within it. Is this interpreted as a concentration of outlying sources associated with the SMC? Alternatively, is only a small fraction of sources actually associated with the Cloud, the rest being background objects?

Mathewson: On closer inspection of the Small Cloud $1410 \mathrm{Mc} / \mathrm{s}$ isophotes you would find a higher concentration of strong sources in the main body of the Cloud than outside. They all lie within 1-2' of arc of an emission region and their $1410 \mathrm{Mc} / \mathrm{s}$ flux densities agree very well with that predicted from $\mathrm{H} \beta$ flux densities. I think it is quite reasonable to associate these objects with the Small Cloud. No suggestion has been made that the outlying radio sources are associated 
with the Clouds. However, it may be worth while for optical astronomers to investigate the regions to the south of the Small Cloud where a cluster of sources with thermal-type spectral indices has been found.

Bok: Have you made a search for sources by methods comparable to those used for the Large and Small Clouds for one or more comparison fields well outside either Cloud, but still in the same part of the sky? It would be of great interest to have a survey of a similar region.

Mathewson: We have surveyed the region between the Clouds at 1410 and $408 \mathrm{Mc} / \mathrm{s}$. An interesting point is that no "thermal" sources, i.e. sources with flat spectral indices, were found. Mr. Milne and Mr. Price have made a survey at 1410 and $408 \mathrm{Mc} / \mathrm{s}$ of the South Polar Cap region (south of $-76^{\circ}$ ) and when their analysis has been completed it should be very interesting to compare the two surveys as you suggest.

Bolton: We have surveyed a large portion of the sky. North of the SMC and over to the LMC there is a larger concentration of nonthermal sources than anywhere else.

de Vaucouleurs: Have you checked whether a nonthermal source on the north side of the LMC coincides with the peculiar galaxy NGC 1947 which is optically reminiscent of NGC 1316 or NGC 5128 ?

Westerlund: If I may answer that question, I asked Dr. Mathewson to look for this object, but he did not detect it.

Hindman: I would like to point out that a large proportion of the thermal regions marked on Mathewson's map lie within the HI isophotes; thus it is possible that these are developing HII regions not yet identified optically.

Westerlund: Henize $\mathrm{N49}$ in the LMC has [OI] $\lambda 6300$ in emission. This supports its classification as a possible supernova remnant.

Mathewson: It is interesting to note that Henize $63 \mathrm{~A}$ which we have identified with a nonthermal radio source has [OI] in emission.

\title{
62. NEUTRAL HYDROGEN IN THE LARGE MAGELLANIC CLOUD
}

\author{
R. X. McGee and Janice A. Multon \\ CSIRO Radiophysics Laboratory, Sydney
}

\section{Introduction}

The neutral hydrogen gas in the Large Magellanic Cloud has been observed with the 14:.5 beam of the Parkes 210-foot telescope and the 48-channel $\mathrm{H}$-line receiver. We wish to present a progress report based on computer reductions of integrated brightness and median radial velocities for some 4200 profiles. The survey is incomplete for some of the southern regions below dec. $-73^{\circ}$. Assisting in the observations were our colleagues, M. W. Sinclair, C. J. Ohlston, and G. H. Trent and the staff at ANRAO, Parkes.

In the previous HI survey of the Large Cloud made with the same receiver and a 2.2 aerial beam, the intensity contours were quite smooth and were centred on a fairly broad maximum near 30 Doradus. The blurring effect of including regions of $2 \mathrm{kpc}$ diameter in an aerial beam of this size was seen in a consistent profile average halfwidth of $50 \mathrm{~km} / \mathrm{sec}$.

The present method of observation was to track the aerial along lines of constant declination at a rate in right ascension equivalent to $3^{\prime}$ of arc per min of time. Thus two $\mathrm{H}$-line profiles were recorded per beamwidth - one profile in each $2 \mathrm{~min}$ of time. 